\title{
SHALON observations of Active Galactic Nuclei at red shift from $z=0.0179$ to $z=1.375$
}

\section{V.G. Sinitsyna*}

P.N. Lebedev Physical Institute, Leninsky pr. 53, Moscow, Russia

E-mail: sinits@sci.lebedev.ru

\author{
A.Y. Alaverdyan, M.S. Andreeva, K.A. Balygin, S.S. Borisov, I.A. Ivanov, \\ A.M. Kirichenko, A.I. Klimov, I.P. Kozhukhova, N.I. Moseiko, S.I. Nikolsky, \\ A.I. Palamarchuk, V.Y. Sinitsyna, I.G. Volokh \\ P.N. Lebedev Physical Institute, Leninsky pr. 53, Moscow, Russia
}

\begin{abstract}
The radio-loud Active Galactic Nuclei (AGNi) having the radio emission arising from a core region rather than from lobes are often referred to as "blazars" and include Flat Spectrum Radio Quasars (FSRQ) and BL Lacertae (BL Lac) objects. We present results of long term observations of FSRQ: among them are known object $3 \mathrm{c} 454.3(\mathrm{z}=0.859)$, high-red shifted quasar $1739+522$ $(4 \mathrm{c}+51.37)(\mathrm{z}=1.375)$ and $4 \mathrm{c}+31.63(\mathrm{z}=0.295), 4 \mathrm{c}+55.17(\mathrm{z}=0.896)$ as well as BL Lac type object OJ $287(\mathrm{z}=0.306)$ that was recently detected by SHALON Cherenkov telescopes. Also, the observation data on known BL Lac objects Mkn 421 ( $\mathrm{z}=0.031)$, Mkn 501 ( $\mathrm{z}=0.034)$, Mkn 180 $(\mathrm{z}=0.046)$ and Radio galaxies NGC $1275(\mathrm{z}=0.018), 3 \mathrm{c} 382(\mathrm{z}=0.0578)$ are summarized in this paper. The observation results are presented with integral spectra, images and spectral energy distributions for each of sources at energies above $800 \mathrm{GeV}$. A number of variability periods in different wavelengths including VHE $\gamma$-rays were found.
\end{abstract}

Frontiers of Fundamental Physics 14 - FFP14,

15-18 July 2014

Aix Marseille University (AMU) Saint-Charles Campus, Marseille

${ }^{*}$ Speaker. 


\section{Introduction}

In the last years, high and very high energy $\gamma$-rays have come to play an important role in the study of Active Galactic Nuclei. A big number of AGNi have been detected through the $\mathrm{MeV}$ to $\mathrm{TeV}$ energies. The long term SHALON observations yielded data on extragalactic sources of different type at energy range of $800 \mathrm{GeV}-100 \mathrm{TeV}$. During the period $1992-2014$ SHALON has been used for observations different type sources[1]. Among them are NGC 1275 ( $\mathrm{z}=0.018$ )(Seyfert); 3c 382 ( $\mathrm{z}=0.0578$ ) (BLRG); known BLLac-type sources: Mkn 421, Mkn 501, Mkn 180, OJ 287; and FSRQs: $1739+522(\mathrm{z}=1.375), 3 \mathrm{c} 454.3(\mathrm{z}=0.859), 4 \mathrm{c}+55.17(\mathrm{z}=0.896), 4 \mathrm{c}+31.63(\mathrm{z}=0.295)$. The representative results on fluxes, images and spectral energy distributions are shown in Figs. 1, 2 and energy spectrum parameters are presented in the table 1 these Proceedings.

\section{NGC $1275(z=0.018)$ and Perseus Cluster}

The results of 15-year observations of the AGN NGC 1275 at energies $800 \mathrm{GeV}-40 \mathrm{TeV}$ discovered by the SHALON telescope in 1996 [2, 3] are presented in Fig. 1. Gamma-ray emission from NGC 1275 was detected by the SHALON at the $31.4 \sigma$. The average integral flux at energies $>800 \mathrm{GeV}$ for NGC 1275 is $(7.8 \pm 0.5) \times 10^{-13} \mathrm{~cm}^{-2} \mathrm{~s}^{-1}$. To analyze the emission related to the core, we identified the emission component corresponding to the central region of NGC 1275 with a size of 32". The spectrum of core emission by SHALON is presented in Fig. 1 left with black triangles. The TeV structure around NGC 1275 (Fig. 1 right) that spatially coincides with the X-ray emission regions can be produced by mechanisms related to the generation of an X-ray structure.

\section{Mkn $421(\mathrm{z}=\mathbf{0 . 0 3 1})$}

The B1 Lac Mkn 421 was detected as the first and the nearest $(\mathrm{z}=0.031)$ metagalactic source of blazar type of TeV energy $\gamma$-quanta in 1992 year using Whipple telescope [4].

Mkn 421 is being intensively studied since 1994 by SHALON [5, 6]. Figure. 1 shows the spectral energy distribution of Mkn 421 by SHALON in comparison with other experiment data from modern and archival observations [7, 8]. The averaged for the whole period of observation since 1994 integral $\gamma$-ray flux above $800 \mathrm{GeV}(0.63 \pm 0.05) \times 10^{-12} \mathrm{~cm}^{-2} \mathrm{~s}^{-1}(16.2 \sigma$ [9]). The increase of the flux over the average value was detected in 1997 and 2004 observations of Mkn 421 by SHALON and estimated to be $(1.01 \pm 0.25) \times 10^{-12} \mathrm{~cm}^{-2} \mathrm{~s}^{-1}$ and $(0.96 \pm 0.2) \times 10^{-12} \mathrm{~cm}^{-2} \mathrm{~s}^{-1}$, respectively. The similar variations of the flux was also observed with the telescopes of Whipple, HEGRA, TACTIC, HESS, MAGIC.

\section{Mkn $501(\mathrm{z}=\mathbf{0 . 0 3 4})$}

In contrast to Mkn 421, Mkn 501 was the first object to be discovered by as $\gamma$-ray source from the ground by Whipple in 1995 [10]. The integral average $\gamma$-ray flux $>800 \mathrm{GeV}$ by SHALON was estimated as $(0.86 \pm 0.06) \times 10^{-12} \mathrm{~cm}^{-2} \mathrm{~s}^{-1}(20.8 \sigma$ [9]). The significant increase of Mkn 501 flux was detected in 1997 and 2006 with the VHE ground telescopes all over the world. The integral $\gamma$-ray flux in 1997 and Aug. 2006 by SHALON telescope was estimated as $(1.21 \pm 0.13) \times$ $10^{-12} \mathrm{~cm}^{-2} \mathrm{~s}^{-1}$ and $(2.05 \pm 0.23) \times 10^{-12} \mathrm{~cm}^{-2} \mathrm{~s}^{-1}$, respectively [6]. The last flaring state of Mkn 501 at the very high energies was detected in the SHALON observational period between March and June 2009. The flux increase was detected at 23-24 April and 23-25 May with average flaring flux of $(3.41 \pm 0.70) \times 10^{-12} \mathrm{~cm}^{-2} \mathrm{~s}^{-1}$ [1]. This increase is correlated with the flaring activity at lower energy range in observations of Fermi LAT [8] and VERITAS, MAGIC, Whipple [8]. 

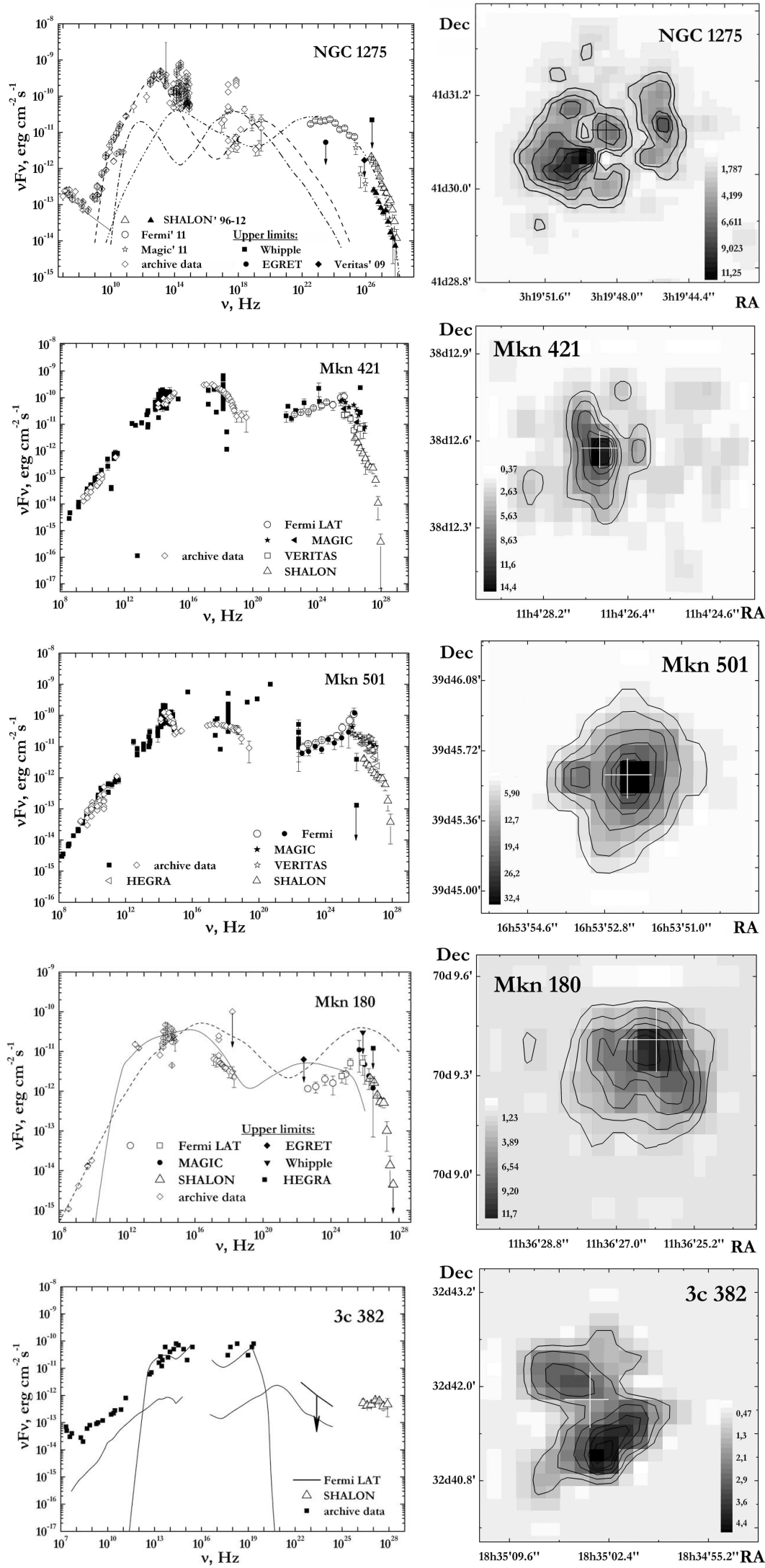

Figure 1: left: Spectral energy distributions of the $\gamma$-ray emission from Active Galactic Nuclei observed by SHALON in comparison with other experiments. The open triangles at TeV energies are SHALON data. right: The images of AGNi in the gamma-rays with energies $>0.8 \mathrm{TeV}$ by SHALON. 

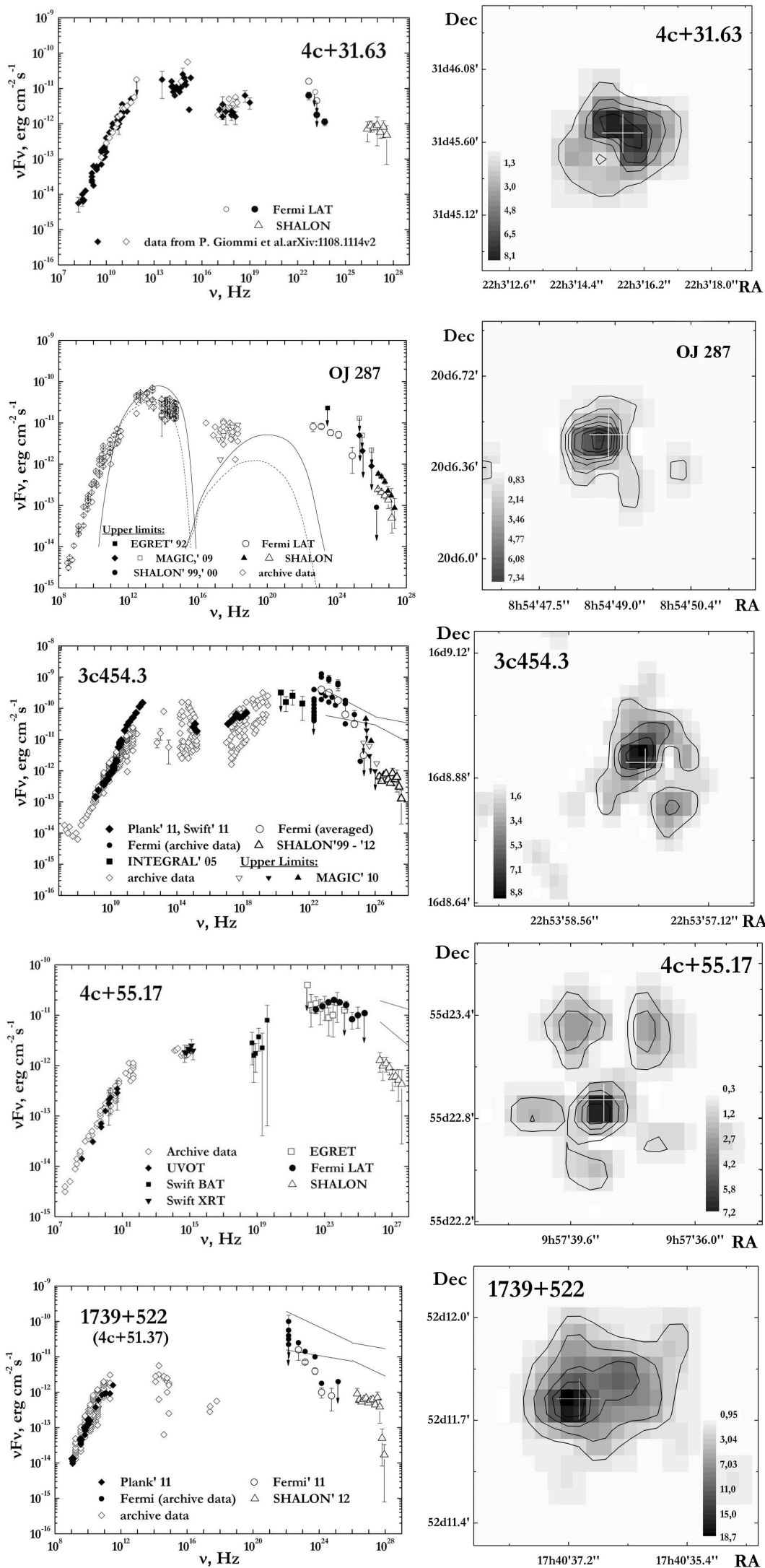

Figure 2: left: Spectral energy distributions of the $\gamma$-ray emission from Active Galactic Nuclei observed by SHALON in comparison with other experiments. The open triangles at TeV energies are SHALON data. right: The images of AGNi in the gamma-rays with energies $>0.8 \mathrm{TeV}$ by SHALON. 


\section{4. $M k n 180(z=0.046)$}

As the VHE $\gamma$-ray emitting Active Galactic Nuclei are variable in flux in all wavebands the correlations between low energy emission (for example X-ray) and $\gamma$-ray emission have been found. Recently, optical - $\mathrm{TeV} / \mathrm{GeV}$ correlation was also found. Mkn 180 was detected in $\mathrm{TeV} \gamma$ rays by MAGIC during an optical high state [11] (Fig. 1 and [12, 13, 14, 15]).

Mkn 180 was observed by SHALON in 2007, 2009 - 2014, for a total of 81 hours. After the standard analysis, a clear excess corresponding to a $18.5 \sigma$ [9]. No evidence for flux variability was found. The observed integral flux above $800 \mathrm{GeV}$ is $(0.65 \pm 0.09) \times 10^{-12} \mathrm{~cm}^{-2} \mathrm{~s}^{-1}$ [1](Fig. 1).

\section{5. $3 c 382(z=0.0578)$}

3c 382 is a typical Broad Line Radio Galaxy (BLRG), essentially identical to a quasar except that the optical luminosity. Its radio structure shows several quasar-like features. The core is very bright and there is a narrow jet with the compact hotspot and with the lobe opposite the jet. In contrast to blazars, the jets in BLRGs are not pointing directly toward the observer, and the relativistic beaming effects and the related jet dominance are only moderate, so it became possible to investigate both the accretion disk and the jet.

3c 382 has been detected by SHALON at TeV energies [16] (in observations of 2009 - 2014 years, 43.1 hours in the total) with a significance [9] of 9.5 $\sigma$. The integral $\gamma$-ray flux above 0.8 $\mathrm{TeV}$ was estimated as $(0.91 \pm 0.14) \times 10^{-12} \mathrm{~cm}^{-2} \mathrm{~s}^{-1}$. The energy spectrum of $\gamma$-rays in the energy region above $800 \mathrm{GeV}$ is described by the power law with the index $-1.08 \pm 0.11$ (Fig. 1). TeV image of $3 c 382$ is correlated with the structures visible in radio energies by VLA [17].

\section{6. $4 c+31.63(z=0.295)$}

In observations of 2012 a new metagalactic source was detected by SHALON in TeV energies (Fig. 2). This object was identified with FSRQ type source $4 c+31.63$ at redshift $z=0.295 .4 c+31.63$ was observed by SHALON in the 2012 - 2014 for a total of 39.3 hours, at zenith angles ranging from $12^{\circ}$ to $35^{\circ}$ [1]. The $\gamma$-ray emission from the position of $4 c+31.63$ was detected above 800 $\mathrm{GeV}$ with a significance[9] of $8.6 \sigma$. The integral $\gamma$-ray flux for this source is found to be $(0.73 \pm$ $0.16) \times 10^{-12} \mathrm{~cm}^{-2} \mathrm{~s}^{-1} .4 \mathrm{c}+31.63$ was previously detected at high energies with Fermi LAT [18].

\section{OJ $287(z=0.306)$}

OJ 287 is an low-frequency peaked BL Lac objects. The most outstanding characteristic of OJ 287 is its 12 year period, which is discovered in optical range [19] and has also been confirmed in the X-ray band. OJ 287 is supposed to be a binary black hole system in which a secondary black hole passes the accretion disk of the primary black hole and produces two impact flashes per period. OJ 287 has been detected with GeV emissions by EGRET [20] and Fermi LAT[21].

OJ 287 was observed by SHALON in 1999, 2000, 2008, 2009 and 2010, for a total of 47.3 hours (for details see [22]).In observations of 2008, 2009 and 2010 (31.2 hours in total) the weak $\gamma$-ray flux was detected [22]. An excess corresponding to a $6.9 \sigma[9]$ was determined. The observed integral flux above $800 \mathrm{GeV}$ is $(0.26 \pm 0.07) \times 10^{-12} \mathrm{~cm}^{-2} \mathrm{~s}^{-1}$. OJ 287 is the weakest extragalactic source observed by SHALON. The flux increase over the detected average flux was detected in 2010 [22] with value of $(0.63 \pm 0.15) \times 10^{-12} \mathrm{~cm}^{-2} \mathrm{~s}^{-1}$ (statistical significance of $6.2 \sigma$ [9]). 
Figure 2 presents spectral energy distribution [23] of the $\gamma$-ray emission from OJ 287 by SHALON [1,22] in comparison with other experiment data and predictions [20, 21, 23] The open triangles at $\mathrm{TeV}$ energies on Fig. 2 are SHALON spectrum of OJ 287; the black triangles present the $\gamma$-ray spectrum at the increased flux period of 2010; an upper limit at $>0.8 \mathrm{TeV}$ corresponds to SHALON observations in 1999, 2000.

\section{8. $3 \mathrm{c} 454.3(\mathrm{z}=0.859)$}

In 1998 year a 3c454.3 ( $\mathrm{z}=0.859)$ had been detected by SHALON at TeV energies [24, 25, 26, $27,28]$. The integral $\gamma$-ray flux above $0.8 \mathrm{TeV}$ was estimated as $(0.43 \pm 0.07) \times 10^{-12} \mathrm{~cm}^{-2} \mathrm{~s}^{-1}$ (14.3 $\sigma$ [9]). Within the range $0.8-9 \mathrm{TeV}$, the observed integral energy spectrum is described by the power law $I\left(>E_{\gamma}\right) \propto E_{\gamma}^{-0.85 \pm 0.07}$. The $\mathrm{TeV} \gamma$-ray emission map is presented in Fig. 2. Recently, $3 c 454.3$ has detected with Fermi LAT at energies $200 \mathrm{MeV}-300 \mathrm{GeV}$ [29].

$3 \mathrm{c} 454.3$ shows the significant flux variability in the different energy ranges including high and very high energies. The last significant flaring state of $3 c 454.3$ at $\mathrm{TeV}$ energies was detected in the SHALON observational period of Nov. - Dec. 2010. The flux increase was detected at 02-04 Dec. with flux of $(3.41 \pm 0.70) \times 10^{-12} \mathrm{~cm}^{-2} \mathrm{~s}^{-1}$. This increase is correlated with the flaring activity at lower energy range in observations of Fermi LAT[30].

\section{9. $4 \mathrm{c}+55.17(\mathrm{z}=\mathbf{0 . 8 9 6})$}

$4 c+55.17$, at redshift $\mathrm{z}=0.896$, is the radio-loud active galaxy classified as a FSRQ. This object was previously detected at high energies with EGRET and Fermi LAT with unusually hard MeV-GeV $\gamma$-ray spectrum [31]. 4c+55.17 was observed by SHALON [1] in the period from 2012 - 2014 at the clear moonless nights, for a total of 49.6 hours, at zenith angles ranging from $13^{\circ}$ to $35^{\circ}$. After the standard analysis, a clear excess corresponding to a $8.4 \sigma$ [9] was determined with integral flux of $I(>0.8 \mathrm{TeV})=(0.90 \pm 0.16) \times 10^{-12} \mathrm{~cm}^{-2} \mathrm{~s}^{-1}$ and the observed integral energy spectrum is fitted by the power law $I\left(>E_{\gamma}\right) \propto E_{\gamma}^{-1.40 \pm 0.15}$.

\section{0. $1739+522(4 c+51.37)(z=1.375)$}

One more remote metagalactic $\gamma$ - source of FSRQ type was detected by SHALON in 1999 and is being intensively studied since then [24, 25, 26, 27, 28]. This object was identified with the active galactic nucleus $1739+522(4 c+51.37)$. This the most distant object (with redshift $\mathrm{z}=1.375$ ) is also the most powerful: its integral $\gamma$-ray flux is found to be $(0.49 \pm 0.05) \times 10^{-12} \mathrm{~cm}^{-2} \mathrm{~s}^{-1}$ $(15.1 \sigma$ [9]) at energies of $>0.8 \mathrm{TeV}$. Within the range $0.8-7 \mathrm{TeV}$, the integral energy spectrum is described by the power law $I\left(>E_{\gamma}\right) \propto E_{\gamma}^{-0.93 \pm 0.09}$. The image of $4 \mathrm{c}+51.37$ at $\mathrm{TeV}$ energies by SHALON is presented at Fig. 2. The high energy $\gamma$-ray emission from $1739+522$ was detected with Fermi LAT in the range $100 \mathrm{MeV}-100 \mathrm{GeV}$ [32].

\section{Conclusion}

The SHALON long-term observations yielded data on extragalactic sources of different type at energy range of $800 \mathrm{GeV}-100 \mathrm{TeV}$. Among them are NGC $1275(\mathrm{z}=0.018), 3 \mathrm{c} 382(\mathrm{z}=0.0578)$, $4 \mathrm{c}+31.63(\mathrm{z}=0.295) ;$ OJ $287(\mathrm{z}=0.306) ; 3 \mathrm{c} 454.3(\mathrm{z}=0.859) ; 4 \mathrm{c}+55.17(\mathrm{z}=0.896) ; 1739+522$ $(\mathrm{z}=1.375)$ which were detected for the first time by SHALON telescope. Also the observation results of known BL Lacs Mkn 421, Mkn 501 and Mkn 180 are obtained. A number of variability periods in different wavelengths including VHE $\gamma$-rays were found. All data from SHALON observations are compared with ones from experiments at high and very high energies. 


\section{References}

[1] V. G. Sinitsyna, V. Y. Sinitsyna, "Astroparticle, Particle, Space Physics and Detectors Physics Applications" (Proc.14th ICATPP, Como, 2013) eds. S Giani, C Leroy, P G Rancoita, L Price and R Ruchti (Singapore: World Scientific) 8 p 3; ibid. p. 20, (2014).

[2] V. G. Sinitsyna, V. Y. Sinitsyna, Astron. Lett. 40(2-3) 75 (2014).

[3] V. G. Sinitsyna, Towards a Major Atmospheric Cherenkov Detector-V, (Kruger Park) ed O C deJager (Potchefstroom: Westprint) p 136 (1997)

[4] M. S. Schubell et. al., Astrophys. J. 460644 (1996).

[5] V. G. Sinitsyna, Nuovo Cim. 19C 965 (1996).

[6] V. G. Sinitsyna et al., J. Phys. Soc. Jpn., Suppl. A 78192 (2009).

[7] A. A. Abdo et al., Astrophys. J. 71630 (2010).

[8] A. A. Abdo et al., Astrophys. J. 727129 (2011).

[9] T.-P. Li, Y.-Q. Ma, Astrophys. J. 272317 (1983).

[10] T. C. Weekes et al., Prep. Ser. 4811 (1999).

[11] J. Albert et al., Astrophys. J. 648 L105 (2006).

[12] D. Horan et al., Astrophys. J. 60351 (2004).

[13] A. A. Abdo et al., Astrophys. J. 7071310 (2009).

[14] L. Costamante, G. Ghisellini, Astron.\&Astrophys. 38456 (2002).

[15] G. Fossati, et al., Astrophys. J. 541166 (2000).

[16] V. G. Sinitsyna, V. Y. Sinitsyna, 2013 EPJ Web of Conferences 5210005.

[17] J. P. Leahy, R. A. Perley, Astrophys. J. 102537 (1991).

[18] P. Giommi et al., Astron.\&Astrophys. 541, A160 (2012)

[19] E. Valtaoja et al., Astrophys. J. 531744 (2000).

[20] R. C. Hartman et al., ApJS 12379 (1999).

[21] A. A. Abdo et al., Astrophys. J. 700597 (2009); Astrophys. J. 715429132 (2010).

[22] V. G. Sinitsyna et al., Proc. 32nd ICRC (Beijing, China), 8/11 26 (2011).

[23] M. Hayashida et al., 2009 Proc. of 31st ICRC, Lodz

[24] V. G. Sinitsyna et al., Int. J. Mod. Phys. A 29, 7023, 7026, 7029 (2005).

[25] V. G. Sinitsyna et al., Nucl. Phys. B (Proc. Suppl.) 196, 251, 442 (2009); ibid. 175-176, 463 (2008); ibid. 151, 108 (2006); ibid. 122, 247, 409 (2003); ibid. 97, 215 and 219 (2001); ibid. 75A, 352 (1999).

[26] S. I. Nikolsky, V. G. Sinitsyna, Phys. Atom. Nucl. 67, 1900 (2004).

[27] V. G. Sinitsyna, AIP (Conf. Proc.) 515, 205 and 293 (2000).

[28] V. G. Sinitsyna et al., Izv. Ross. Akad. Nauk Ser. Fiz. 71(7), 94 (2007).

[29] A. A. Abdo et al., Astrophys. J. 699, 817 (2009).

[30] A. A. Abdo et al., Astrophys. J. 733, L26 (2011).

[31] W. McConville et al., Astrophys. J. 738, 48 (2011).

[32] P. L. Nolan et al., ApJS 19931 (2012). 